\title{
Angiogenic Potential of Gellan-Gum-Based Hydrogels for Application in Nucleus Pulposus Regeneration: In Vivo Study
}

\author{
Joana Silva-Correia, Ph.D.,,2 Vera Miranda-Gonçalves, B.Sc., ${ }^{2,3}$ António J. Salgado, Ph.D., ${ }^{2,3}$ \\ Nuno Sousa, M.D., Ph.D., ${ }^{2,3}$ Joaquim M. Oliveira, Ph.D., ${ }^{1,2}$ Rui M. Reis, Ph.D., ${ }^{2-4}$ and Rui L. Reis, Ph.D. ${ }^{1,2}$
}

Hydrogels for nucleus pulposus (NP) regeneration should be able to comprise a nonangiogenic or even antiangiogenic feature. Gellan gum (GG)-based hydrogels have been reported to possess adequate properties for being used as NP substitutes in acellular and cellular strategies, due to its ability to support cell encapsulation, adequate mechanical properties, and noncytotoxicity. In this study, the angiogenic response of GG-based hydrogels was investigated by performing the chorioallantoic membrane assay. The convergence of macroscopic blood vessels toward the GG, ionic-crosslinked methacrylated GG (iGG-MA), and photo-crosslinked methacrylated GG (phGGMA) hydrogel discs was quantified. Gelatin sponge (GSp) and filter paper (FP) alone and with vascular endothelial growth factor were used as controls of angiogenesis. The images obtained were digitally processed and analyzed by three independent observers. The macroscopic blood vessel quantification demonstrated that the GG-based hydrogels are not angiogenic as compared with FP controls. No statistical differences between the GG-based hydrogels tested in respect to its angiogenic ability were observed. Hematoxylin and eosin staining and SNA-lectin immunohistochemistry assay indicated that the iGG-MA and phGG-MA hydrogels do not allow the ingrowth of chick endothelial cells, following 4 days of implantation. On the contrary, GG, GSp, and FP controls allowed cell infiltration. The histological data also indicated that the GG-based hydrogels do not elicit any acute inflammatory response. The results showed that the GG, iGG-MA, and phGG-MA hydrogels present different permeability to cells but functioned as a physical barrier for vascular invasion. These hydrogels present promising and tunable properties for being used as NP substitutes in the treatment of degenerative intervertebral disc.

\section{Introduction}

$\mathbf{N}$ ATURAL-BASED HYDROGELS have been receiving considerable attention in the last decade for application in tissue engineering of nucleus pulposus (NP). ${ }^{1,2}$ Biomaterials based on alginate, ${ }^{3,4}$ hyaluronic acid, ${ }^{5}$ carboxymethylcellulose, ${ }^{6}$ collagen, ${ }^{7}$ or chitosan ${ }^{8,9}$ are being investigated as potential NP scaffolds, mainly due to their similarity to the native tissue in terms of structural, viscoelastic, and mechanical properties. Other advantageous characteristics include their intrinsic noncytotoxicity, easiness of use for minimum-invasive procedures, and good cell adhesiveness and integration.

Gellan gum (GG), which is an extracellular polysaccharide secreted by Sphingomonas elodea, forms a firm and transparent gel with interesting features for use as an in vitro three- dimensional (3D) cell support, or as an in vivo injectable system. ${ }^{10}$ Previous studies have demonstrated the therapeutic potential of GG in the cartilage tissue engineering field. ${ }^{11,12}$ The GG hydrogel was shown to efficiently sustain the deliver and growth of human articular chondrocytes by in situ gelation, and support the deposition of a hyaline-like extracellular matrix, leading to the formation of a functional cartilage tissue-engineered construct. In a recent work, formulations of low-acyl and high-acyl GG have been processed as microparticles/matrices systems for application as NP substitutes. ${ }^{13}$ This new system provides enhanced control over the mechanical properties of the hydrogel (by mixing the two forms of GG at different ratios), while allowing an efficient cellular encapsulation and distribution. Despite its interesting physicochemical properties and noncytotoxicity, the material presents some limitations, namely, the limited

\footnotetext{
${ }^{1}$ Department of Polymer Engineering, 3B's Research Group-Biomaterials, Biodegradables and Biomimetics, University of Minho, Headquarters of the European Institute of Excellence on Tissue Engineering and Regenerative Medicine, Guimarães, Portugal.

${ }^{2}$ ICVS/3B's-PT Government Associate Laboratory, Braga/Guimarães, Portugal.

${ }^{3}$ Life and Health Science Research Institute (ICVS), School of Health Sciences, University of Minho, Braga, Portugal.

${ }^{4}$ Molecular Oncology Research Center, Barretos Cancer Hospital, Barretos, São Paulo, Brazil.
} 
control of structural properties for longer periods of time. GG-based hydrogels, such as the ionic-crosslinked methacrylated GG (iGG-MA) and photo-crosslinked methacrylated GG (phGG-MA) hydrogels, have been recently proposed as potential alternative implants for NP regeneration. ${ }^{14,15}$ In this previous work, we have combined the most advantageous characteristics of GG with the possibility of tuning the polymerization process, both temporally and spatially, which can be induced by immersion in a saline solution at physiological $\mathrm{pH}$ and/or by exposure to UV light. This system was produced by reacting low-acyl GG with glycidyl methacrylate (GMA) at $\mathrm{pH} 8.5$ in order to enable the incorporation of methacrylate groups in GG structure. The final product, that is, methacrylated gellan gum (GG-MA), can be easily dissolved in water at room temperature. By adjusting the reaction parameters, it was also possible to have a limited control over the final structural and mechanical properties of the hydrogel. The physicochemical and in vitro biological characterization of the iGG-MA and phGG-MA hydrogels demonstrated that these hydrogels were stable in a phosphate-buffered saline solution $(1 \times ; \mathrm{pH} 7.4)$ for several months, possess improved mechanical properties, and were noncytotoxic. The dynamic mechanical analysis of these hydrogels revealed that the photo-crosslinked material presented higher values for the storage modulus, when compared with GG hydrogels, which is consistent with a higher crosslinking density. ${ }^{14,15}$ By its turn, the ionic-crosslinked GG-MA hydrogels presented an intermediate mechanical behavior. The data suggest that the proposed GG-based hydrogels assemble several characteristics that are promising for NP regeneration, as acellular or cellular substitutes.

The NP is a naturally avascular tissue. ${ }^{1}$ During intervertebral disc degeneration, several tissue changes occur. ${ }^{16}$ Angiogenesis is one of the factors that seem to enhance intervertebral disc degeneration and pain. ${ }^{17}$ Therefore, for an effective function, the NP substitute must both prevent vascular invasion and maintain the typical phenotype of NP cells, without a blood supply.

In this work, our aim was to investigate in vivo the angiogenic/antiangiogenic potential of the GG, iGG-MA, and phGG-MA hydrogels, using an optimized adaptation of the chick embryo chorioallantoic membrane (CAM) assay. ${ }^{18}$ The CAM assay has been used as a model system to study and visualize in vivo human tumor angiogenesis and cell metastasis. ${ }^{19,20}$ It has been also performed to evaluate drug delivery systems ${ }^{21}$ and test the stimulation/inhibition effect of several natural and synthetic compounds on angiogenesis. ${ }^{18}$ Contrarily to the other classical assays for studying angiogenesis in vivo, the CAM assay is simple, feasible for numerous samples, and reliable test. Moreover, it does not require an animal facility, and is not therefore expensive. This method has been also applied to study the angiogenic and inflammatory response to biomaterials. ${ }^{22,23}$ To investigate the angiogenic properties of the GG-based materials, hydrogel discs made of GG, ionic-, and photo-crosslinked GG-MA were placed on the CAM. The macroscopic blood vessel convergence was evaluated in ovo and ex ovo, after 4 days of implantation. Hematoxylin and eosin (H\&E) staining was performed in order to investigate possible blood microvessel formation, inflammation, and cell ingrowth. In addition, immunohistochemical analysis with SNA-lectin specific for chicken endothelial cell staining was performed.

\section{Materials and Methods}

\section{Materials}

For the preparation of GG-based hydrogel discs, low-acyl GG (Gelzan $\left.{ }^{\mathrm{TM}} \mathrm{CM}, F_{w}=1,000,000\right)$, GMA (97\%), and methyl benzoylformate (MBF, 98\%) were purchased from SigmaAldrich Co. For performing the evaluation of the angiogenic effect on the GG-based materials, the following materials and reagents were used: gelatin sponge Cutanplast ${ }^{\circledR}$ (gelatinous and absorbent sponge with haemostatic effect; Mascia Brunelli Spa), filter paper MN GF-3 (Macherey-Nagel), recombinant human VEGF165 (VEGF; Peprotech), H\&E (Merck), and biotinylated Sambucus nigra (Elderberry) bark lectin (SNA, EBL; Vector Laboratories). All other reagents were obtained from Sigma-Aldrich Co., unless otherwise indicated.

\section{Synthesis of GG-MA}

GG-MA was synthesized by reacting GG with GMA as previously reported by Silva-Correia. ${ }^{14,15}$ Briefly, a solution of GG at $1 \%(\mathrm{w} / \mathrm{v})$ final concentration was prepared in distilled water at room temperature and under constant stirring. The solution was heated at $90^{\circ} \mathrm{C}$ in order to obtain the complete and homogeneous dispersion of the material. The GG solution was allowed to cool down until reaching room temperature, and then GMA was added at 20-fold molar excess in respect to the repeating unit of GG. The $\mathrm{pH}$ of the reaction mixture was adjusted to 8.5 using $1 \mathrm{M}$ sodium hydroxide $(\mathrm{NaOH}$; Panreac Química SAU). The reaction occurred for the period of $24 \mathrm{~h}$ at room temperature under vigorous agitation. An automatic adjustment of $\mathrm{pH}$ to 8.5 was carried out using an automatic pump (Fusion 200; Chemyx, Inc.) with $1 \mathrm{M} \mathrm{NaOH}$. The reaction products were precipitated with 0.5 volumes of cold acetone (Panreac Química SAU) and purified by dialysis (cellulose membrane, MW cut-off $12 \mathrm{kDa}$; Sigma) against distilled water for 7 days to remove residual GMA. The purified GG-MA was frozen at $-80^{\circ} \mathrm{C}$ and the powders were obtained after lyophilization (Cryodos -80; Telstar) for 7 days.

\section{Preparation of the GG, iGG-MA, and phGG-MA hydrogel discs}

Prior to the preparation of the hydrogel discs, the low-acyl and GG-MA powders were sterilized under an ethylene oxide gas atmosphere. The materials were processed in a laminar-flow hood using previously sterilized supplies and reagents. All discs were produced with a diameter of $6 \mathrm{~mm}$ and height of $\sim 3 \mathrm{~mm}(100 \mu \mathrm{L} /$ disc $)$.

GG hydrogel discs were produced by dissolving sterile GG powder in distilled water under constant agitation to obtain a final concentration of $2 \%(\mathrm{w} / \mathrm{v})$. The solution was then gradually heated to $90^{\circ} \mathrm{C}$ and maintained at this temperature for $30 \mathrm{~min}$. Following a progressive temperature decrease to $60^{\circ} \mathrm{C}-65^{\circ} \mathrm{C}$, phosphate-buffered saline $1 \times(\mathrm{PBS}, \mathrm{pH} 7.4$; Sigma $)$ solution was added to a final concentration of $10 \%(\mathrm{v} / \mathrm{v})$ in GG solution, after which temperature was continuously decreased to $50^{\circ} \mathrm{C}$. GG discs were produced by transferring the solution to a silicon mould and allowing gelation to occur at room temperature for $\sim 5 \mathrm{~min}$. Afterward, the discs were equilibrated by immersion in PBS ( $\mathrm{pH}$ 7.4) until further use.

The iGG-MA hydrogel discs were obtained by dissolving the sterile GG-MA powder in distilled water at a final concentration of $2 \%(\mathrm{w} / \mathrm{v})$, under vigorous agitation and at room 
temperature. The gel was transferred to a silicon mould and discs were obtained by immersion in PBS (pH 7.4) for $30 \mathrm{~min}$.

The phGG-MA hydrogel discs were produced by using a sterile GG-MA solution at $2 \%(\mathrm{w} / \mathrm{v})$ concentration. After complete dissolution of the GG-MA powder, the photoinitiator MBF was then added to a final concentration of $0.1 \%$ $(\mathrm{w} / \mathrm{v})$ in solution. The gel was transferred to a silicon mould and the hydrogel discs were obtained by exposure to ultraviolet light (366 nm, UV lamp Triwood 6/36; Bresciani srl., Italy) for $10 \mathrm{~min}$. Finally, the phGG-MA discs were further equilibrated in PBS ( $\mathrm{pH} 7.4$ ) for $30 \mathrm{~min}$.

\section{Chick embryo CAM assay}

White fertilized chicken eggs $(n=90-120$; Pintobar, Portugal) were incubated at $37^{\circ} \mathrm{C}$ (Laboratory Incubator Series 8000; Termaks) for 3 days. After this period of time, a small hole was created in the pointed end of the egg to allow dissociation of the CAM from the egg shell membrane. In addition, a circular window (maximum of $15 \mathrm{~mm}$ in diameter) was made into the shell, with the aid of small dissecting scissors, in order to evaluate embryo viability and allow access to the CAM (Fig. 1A). This step was performed in a laminar-flow hood to minimize the risk of contamination. The opening in the shell was then sealed with transparent tape $(\sim 50 \times 30 \mathrm{~mm}$; BTK) to prevent dehydration and the eggs were returned to the incubator at $37^{\circ} \mathrm{C}$ until day 10 of embryonic development.

The sterile hydrogel discs $(n=10)$ were implanted on the CAM at day 10 of embryonic development under sterile conditions. Three experimental groups were tested: GG, iGG-MA, and phGG-MA hydrogel discs. Control groups were also implanted on the CAM at day 10 of embryonic development $(n=10)$, which were based either on filter paper $(\mathrm{FP})$ or gelatin sponge (GSp). FP and GSp discs with a diameter of $6 \mathrm{~mm}$ were prepared and sterilized by autoclave or under an ethylene oxide atmosphere, respectively. The sterile FP and GSp discs were then placed directly on the CAM and used as controls with or without the addition of $100 \mathrm{ng}$ of vascular endothelial growth factor (VEGF) (FP+VEGF and GSp + VEGF). The shell windows were then protected again with transparent tape and the eggs were returned to the incubator at $37^{\circ} \mathrm{C}$ until day 14 of embryonic development. After 2 days, $100 \mathrm{ng}$ of VEGF was added to the FP and GSp VEGF-containing controls, under sterile conditions. The images of the CAM implants were acquired in ovo by the end of the assay using an Olympus DP71 digital camera (Olympus) connected to the Cell ${ }^{\mathrm{B}}$ basic imaging software (Olympus) attached to a stereomicroscope Olympus SZX16 (Olympus). The embryos and their membranes were then fixed in ovo by adding a small volume of freshly prepared paraformaldehyde (PFA; Merck) solution at $4 \%(\mathrm{v} / \mathrm{v})$, followed by incubation at $-80^{\circ} \mathrm{C}$ for $10 \mathrm{~min}$. The implants and the underlying and immediately adjacent CAM portions were cut by means of using forceps and a small dissecting scissor. Then, CAM portions were transferred to 12-well plates containing PFA solution at $4 \%(\mathrm{v} / \mathrm{v})$. Ex ovo images were then captured for each CAM implant directly from the plate. The excised membranes were transferred to histological cassettes, embedded in paraffin, and serially sectioned (Rotary Microtome HM 325; Microm) for immunohistochemical analysis. Three independent CAM assays were performed.

Analysis of blood vessel convergence. The macroscopic evaluation of the angiogenic response was carried out using a semiquantitative method by analyzing the convergence of blood vessels toward the implanted discs both in ovo and ex ovo, as previously described by Ribatti. ${ }^{18}$ The in ovo and ex ovo images obtained at day 14 of embryonic development were processed using the WCIF ImageJ software program (US National Institutes of Health) for facilitating the counting of the total number of blood vessels converging toward the implanted disc. For quantification purposes, the magnification of the stereomicroscope images was kept constant $(7 \times)$, as well as the image-processed area $(1000 \times 1000$ pixels $)$. The total number of macroscopic microvessels that converged toward the graft was counted blindly for each egg by three independent observers.

H\&E staining. The CAM 3- $\mu$ m-thick sections were stained with H\&E. The histological sagital sections of the samples were observed under transmitted microscopy using an Axio Imager.Z1m light microscope (Zeiss) with an attached digital camera AxioCam MRc5 (Zeiss) connected to the AxioVision image processing software (Zeiss).

Immunohistochemical analysis. Representative 3- $\mu \mathrm{m}$ thick CAM sections containing the polymer were subjected to immunohistochemical analysis according to the streptavidin-biotin peroxidase complex system (UltraVision Large Volume Detection System Anti-Polyvalent, HRP; LabVision Corporation). Briefly, paraffin was eliminated off from CAM sections. Then, rehydrated slides were submitted to heat-induced antigen retrieval with $10 \mathrm{mM}$ citrate buffer ( $\mathrm{pH}$ 6.0; Merck) for the period of $20 \mathrm{~min}$ at $98^{\circ} \mathrm{C}$. To inactivate endogenous peroxidases, the slides were incubated in a 3\% (v/v) hydrogen peroxide (Panreac Química SAU) solution for $10 \mathrm{~min}$, and then soaked in PBS. CAM sections were incubated with protein blocking solution for $10 \mathrm{~min}$ followed by incubation with the primary antibody raised against lectin (SNA, EBL) for $1 \mathrm{~h}$ at room temperature. Finally, sections were sequentially washed with PBS and incubated with the streptavidin-peroxidase complex for $10 \mathrm{~min}$. The immune reaction was visualized by $3,3^{\prime}-$ diamonobenzidine (DAB; Thermo Scientific) as a chromogen. All sections were counterstained with Gill-2 hematoxylin (Merck). For preparing the negative controls, the primary antibody was omitted, whereas for positive control the CAM alone was used. The images of the CAM histological sections were acquired using an AxioCam MRc5 digital camera (Zeiss) connected to the AxioVision image processing software (Zeiss) attached to an Axio Imager.Z1m light microscope (Zeiss).

\section{Statistical analysis}

One-way analysis of variance (Tukey's multiple comparison test) was carried out to analyze the statistical differences using the GraphPad Prism software (GraphPad Software, Inc.). Statistical significance was defined at $p<0.05$ for a $95 \%$ confidence interval ( $p$ value: ${ }^{* *}<0.001 ;{ }^{* *}<0.01{ }^{*}<0.05$ ).

\section{Results}

\section{Chick embryo CAM assay}

In this study, the ability of biomaterials in inducing blood vessel formation was investigated by means of performing 


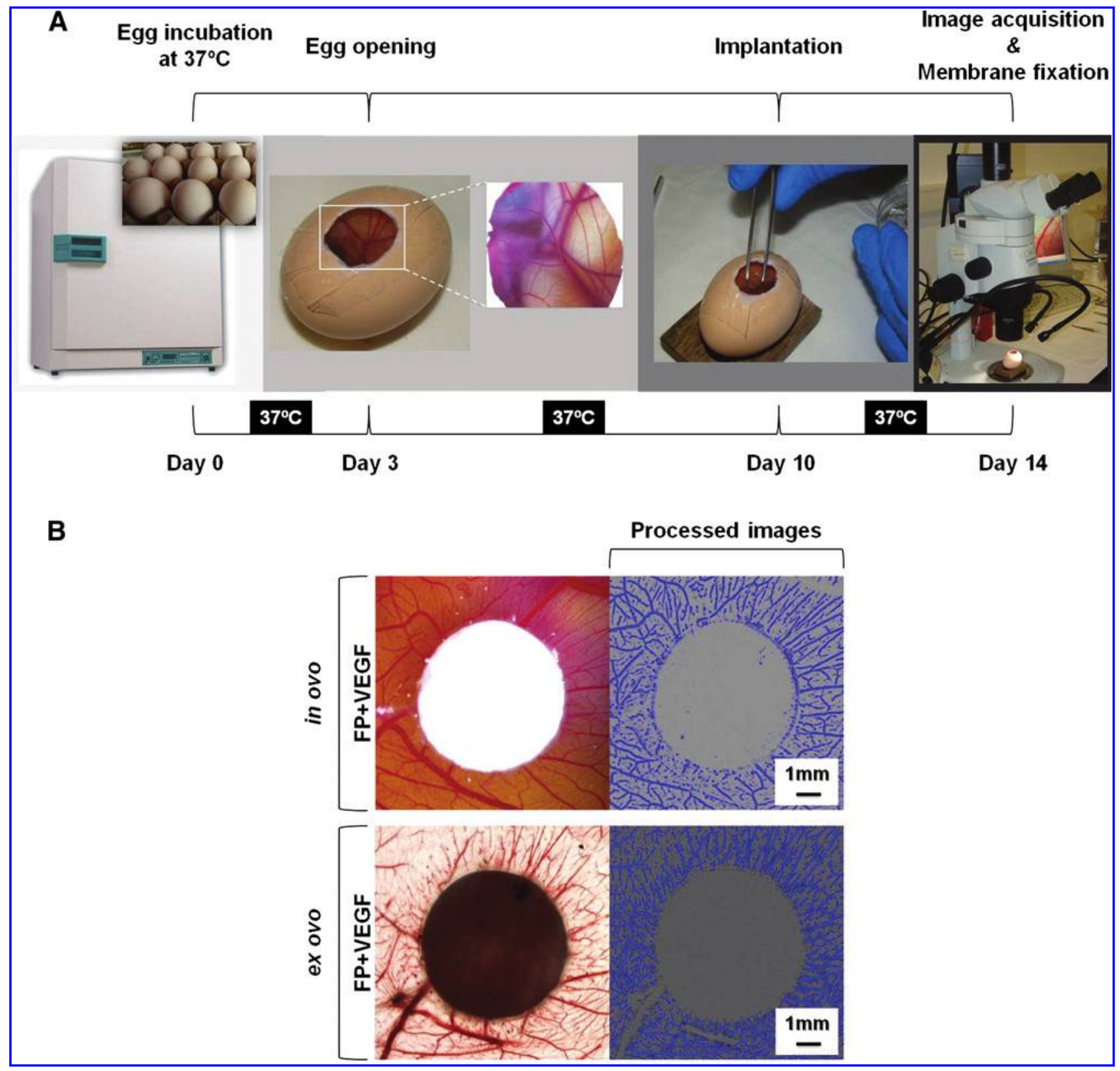

FIG. 1. Schematic representation of the optimized chorioallantoic membrane (CAM) assay (A) and illustrative images of the filter paper (FP) + vascular endothelial growth factor (VEGF) implants, acquired in ovo and ex ovo after 4 days of implantation and respective imaging processing $(\mathbf{B})$. The sterile gellan-gum-based discs and controls were implanted on the CAM of fertilized eggs (previously incubated at $37^{\circ} \mathrm{C}$ ) at day 10 of embryonic development and the assay proceeded until day 14 . The images were acquired in ovo and ex ovo and the membranes were treated for immunohistochemical analysis. The images were processed for determining macroscopic blood vessel convergence. Color images available online at www.liebertonline.com/tea

the CAM method at day 10 of embryonic development (Fig. 1). The procedure for exposing, accessing the chick CAM, and the implantation of the materials at day 10 is shown in Figure 1A. The typical stereomicroscopy images of the implants were acquired ex ovo and in ovo, as exemplified in Figure 1B for the FP+VEGF control. The images were processed using the WCIF ImageJ software for determining blood vessel convergence. Figure 2 shows the stereomicroscopy photographs (ex ovo) of the excised CAM sections corresponding to the implanted materials. The corresponding in ovo images are shown as supplementary data (Supplementary Fig. S1; Supplementary Data are available online at www.liebertonline.com/tea). The GG, iGG-MA, and phGG-MA hydrogel discs were produced with a typical diameter of $6 \mathrm{~mm}$. From images, it is possible to observe that a partial absorption of the GG-based hydrogel discs has occurred (Fig. 2A-C). By its turn, it is also possible to observe by the end of the assay that the extent of degradation of control gelatin-based (GSp) group (with and without VEGF stimulation) (Fig. 1D, E) was higher as compared with GGbased hydrogel discs. There is no evidence of absorption of the FP control group (with and without VEGF stimulation) (Fig. 2F, G). Actually, Supplementary Figure S1 also clearly shows that the phGG-MA hydrogels are less absorbed by the 


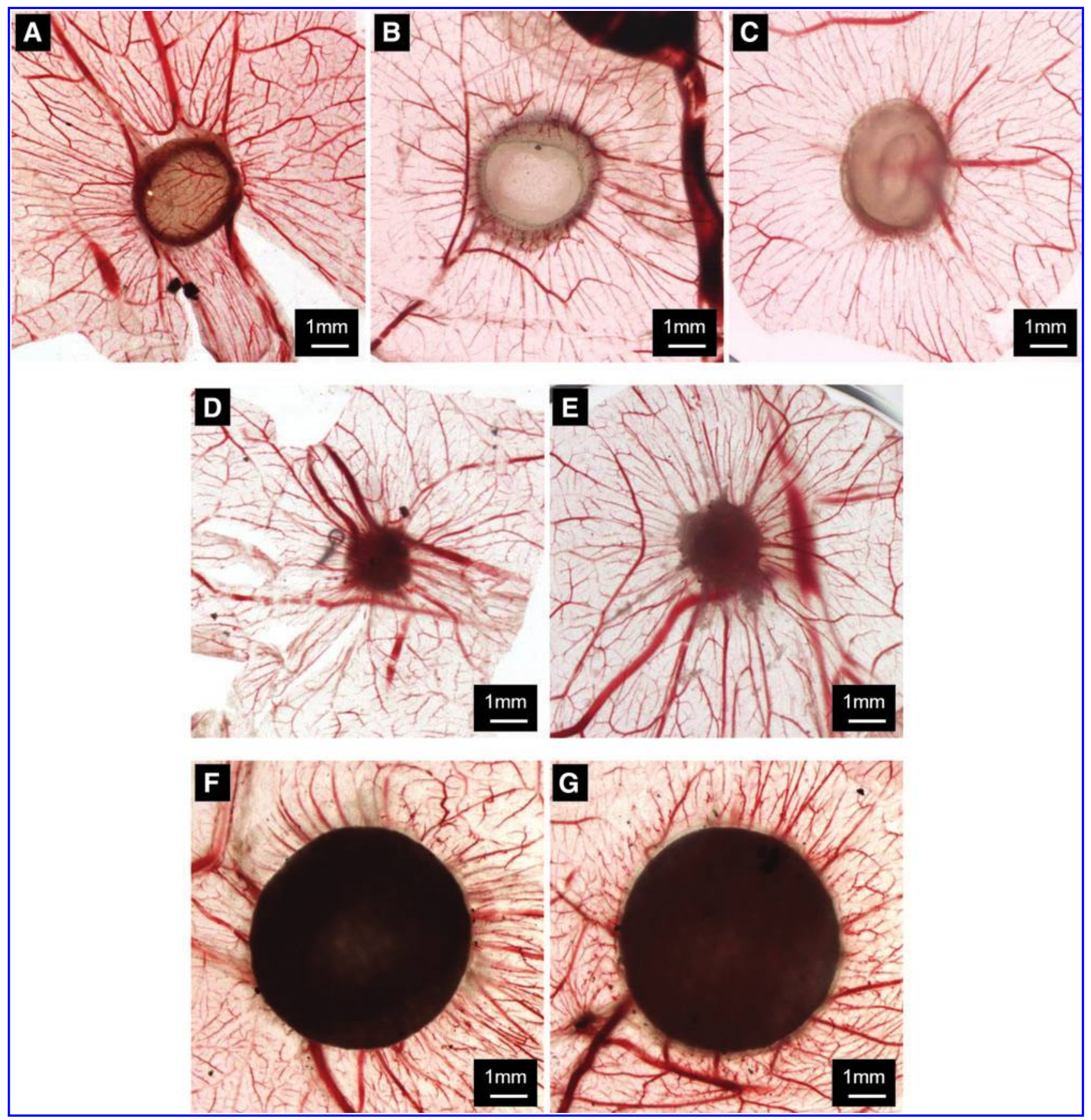

FIG. 2. Stereomicroscopy photographs (ex ovo) of the excised CAM sections corresponding to the implanted materials, after 4 days of implantation: (A) gellan gum (GG), (B) ionic-crosslinked methacrylated gellan gum (iGG-MA), (C) photo-crosslinked methacrylated gellan gum (phGG-MA), (D) gelatin sponge (GSp), (E) GSp + VEGF, (F) FP, and (G) FP+VEGF. The implanted materials were produced with a typical diameter of $6 \mathrm{~mm}$. A partial absorption of the GG-based hydrogel discs $(A-C)$ and control GSp-based group (D and E) is clearly observable by the end of the assay. Color images available online at www.liebertonline.com/tea

CAM as compared with the other hydrogels and GSp group, which is an indication of its superior stability.

\section{Quantification of blood vessels}

The macroscopic evaluation of the angiogenic response was carried out semiquantitatively. The convergence of blood vessels toward the implanted materials was investigated both in ovo and ex ovo (Fig. 3). The quantification of the macroscopic blood vessels converging toward the implanted materials, after 4 days of implantation, was firstly counted from in ovo processed images (Fig. 3A). It can be seen that there are no statistically significant differences in respect to the number of macroscopic blood vessels converging to the GG-based hydrogel discs (GG, iGG-MA, and phGG-MA) and GSp controls (with and without VEGF stimulation), but there is a significantly higher number of macroscopic blood vessels converging to the FP controls (with and without 


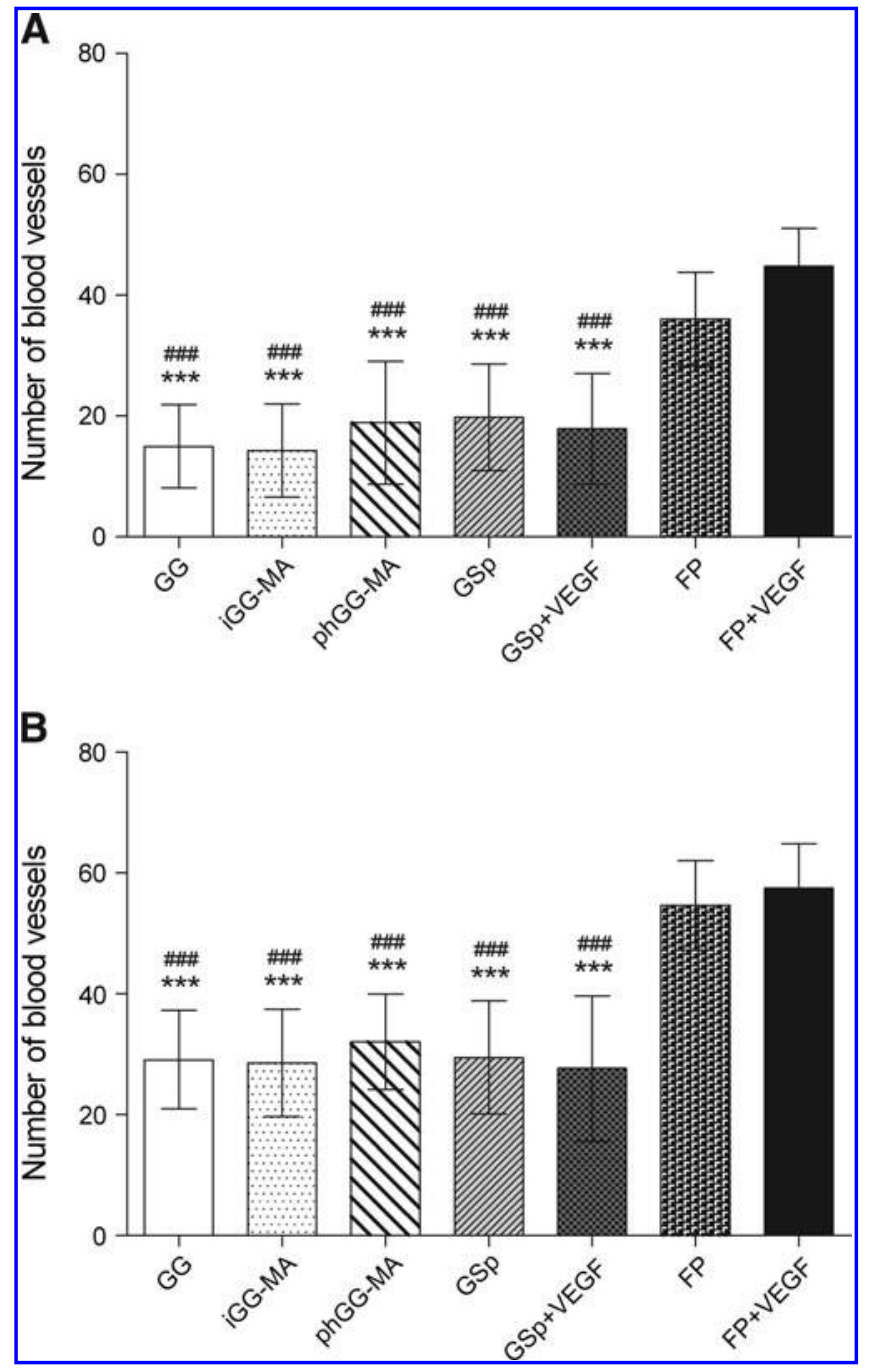

FIG. 3. In ovo (A) and ex ovo (B) quantification of the macroscopic blood vessels converging toward the implanted materials, after 4 days of implantation. The mean number of blood vessels results from the counts performed by three independent observers of a minimum of 10 discs per implant (results are expressed as mean $\pm S D ; n=10$ ). One-way analysis of variance followed by Tukey's multiple comparison test was used for statistical analysis $(* * * p<0.001$ vs. FP, $\#$ \#\# $p<0.001$ vs. FP + VEGF).

VEGF) $\left({ }^{* * *} p<0.001\right.$ vs. FP, ${ }^{\# \# \#} p<0.001$ vs. FP+VEGF). It can also be seen that the stimulation with VEGF, particularly in $\mathrm{FP}$, seems to increase the number of macroscopic blood vessels converging to the material, but these differences are not statistically significant. On the other hand, the addition of VEGF to GSp did not affect the number of the macroscopic and convergent blood vessels.

The number of blood vessels was also counted ex ovo (Fig. $3 \mathrm{~B})$. This data corroborates the results obtained for the in ovo quantification of macroscopic blood vessels. Despite, it can be observed that an overall increase in the number of macroscopic blood vessels as compared with the number of vessels observed in in ovo images. This data shows that the ex ovo identification and quantification of macroscopic blood vessels is facilitated.

\section{H\&E staining and immunohistochemistry}

Histological evaluation can provide useful qualitative information to studies on angiogenesis, particularly for the microscopic identification of blood vessels. Figure 4 shows the light microscopy images of the excised CAM sagital sections with the implants stained with H\&E, after 4 days of implantation. As evidenced in in ovo and ex ovo observations, it can be stated that the GG-based hydrogel discs (Fig. 4A-C) were less absorbed as compared with the GSp group (Fig. $4 \mathrm{D}, \mathrm{E})$. No evidence of absorption was observed for the FP group (Fig. 4F, G). From high-magnification images, it can be seen that GG allows cell infiltration (black arrows), but no signs of cell ingrowth were observed for both iGG-MA and phGG-MA hydrogels. By its turn, GSp and GSp + VEGF were massively colonized by cells. Moreover, an increase in cell density at the interface of CAM/FP controls and in the number of infiltrated cells was observed.

Several macro- and microscopic blood vessels can be observed in the CAM of all implants. Macroscopically, there were no significant differences on the number of blood vessels converging to the GG-based hydrogels and GSp controls (Fig. 3A, B), but a higher number of macroscopic blood vessels in FP + VEGF can be clearly observed. Moreover, a higher density of microscopic blood vessels was observed in the CAM supporting the FP, FP + VEGF, and GSp + VEGF as compared with the others. From Figure $4 \mathrm{~A}-\mathrm{C}$, it can also be observed that phGG-MA presented a higher number of microscopic blood vessels as compared with iGG-MA and GG. The formation of granulation tissue was not observed for all the GG-based hydrogels, which shows that the materials do not elicit any acute inflammatory response.

Complementarily, immunohistochemical analysis of the blood vessels of chick origin at CAM sections was performed with SNA-lectin (Fig. 5). Figure 5A shows that infiltrated cells in the GG hydrogel were stained with SNA-lectin, which specifically binds to chick endothelial cells (brown staining). Figure $5 \mathrm{~A}-\mathrm{C}$ shows that a monolayer of endothelial cells was contacting with the GG-based hydrogel discs at the interface of CAM/hydrogel, but only GG allowed cell ingrowth. Conversely, GSp and GSp + VEGF were massively colonized by different types of cells of chick origin, including endothelial cells (Fig. 5D, E). From Figure 5F and G, it is possible to observe that there is a thicker layer of endothelial cells contacting with the FP control. In fact, a massive group of endothelial cells migrated to the FP implant.

A higher number of microscopic blood vessels was observed in the GSp + VEGF as compared with the GSp. The same trend was observed for the FP controls; that is, stimulation with VEGF allowed to increase the number of endothelial cells at the CAM. This result shows that VEGF allowed recruiting a higher number of endothelial cells to both periphery and core of the control materials, as revealed by labeling with SNA-lectin.

\section{Discussion}

The current studies dealing with natural-based materials for NP regeneration ${ }^{3-9}$ are mainly concerned in producing a $3 \mathrm{D}$ structure that mimics the properties of the native tissue, in terms of structural integrity, hydration, and ability to withstand mechanical loads. These features are evidently essential to achieve the perfect environment for synthesis of new 


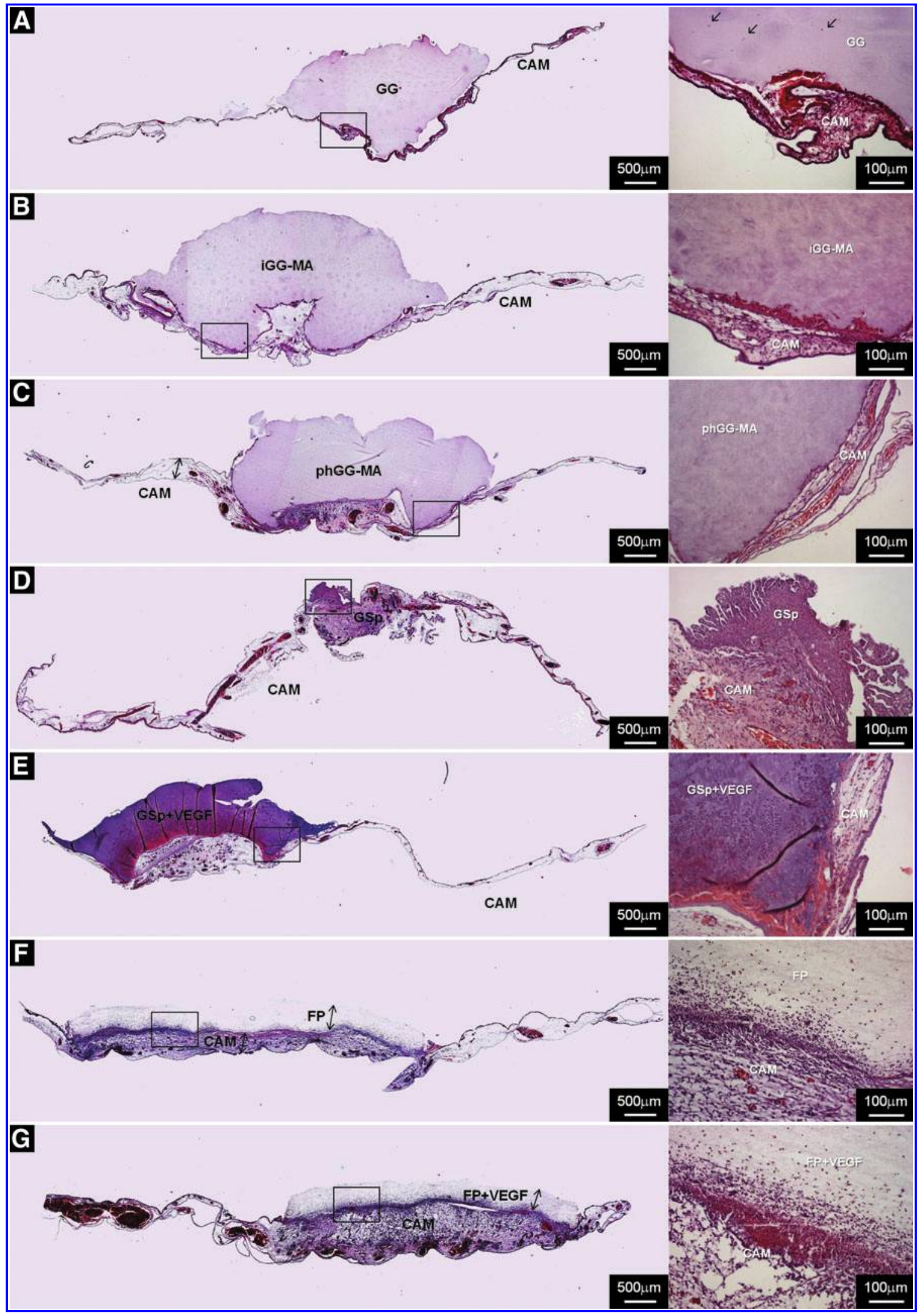

FIG. 4. Light microscopy photographs of the excised CAM sagital sections stained with hematoxylin and eosin (H\&E) corresponding to the implanted materials, after 4 days of implantation: (A) GG, (B) iGG-MA, (C) phGG-MA, (D) GSp, (E) GSp + VEGF, (F) FP, and (G) FP + VEGF. The arrows indicate infiltrating cells and squares represent the region corresponding to the magnified images. Color images available online at www.liebertonline.com/tea 


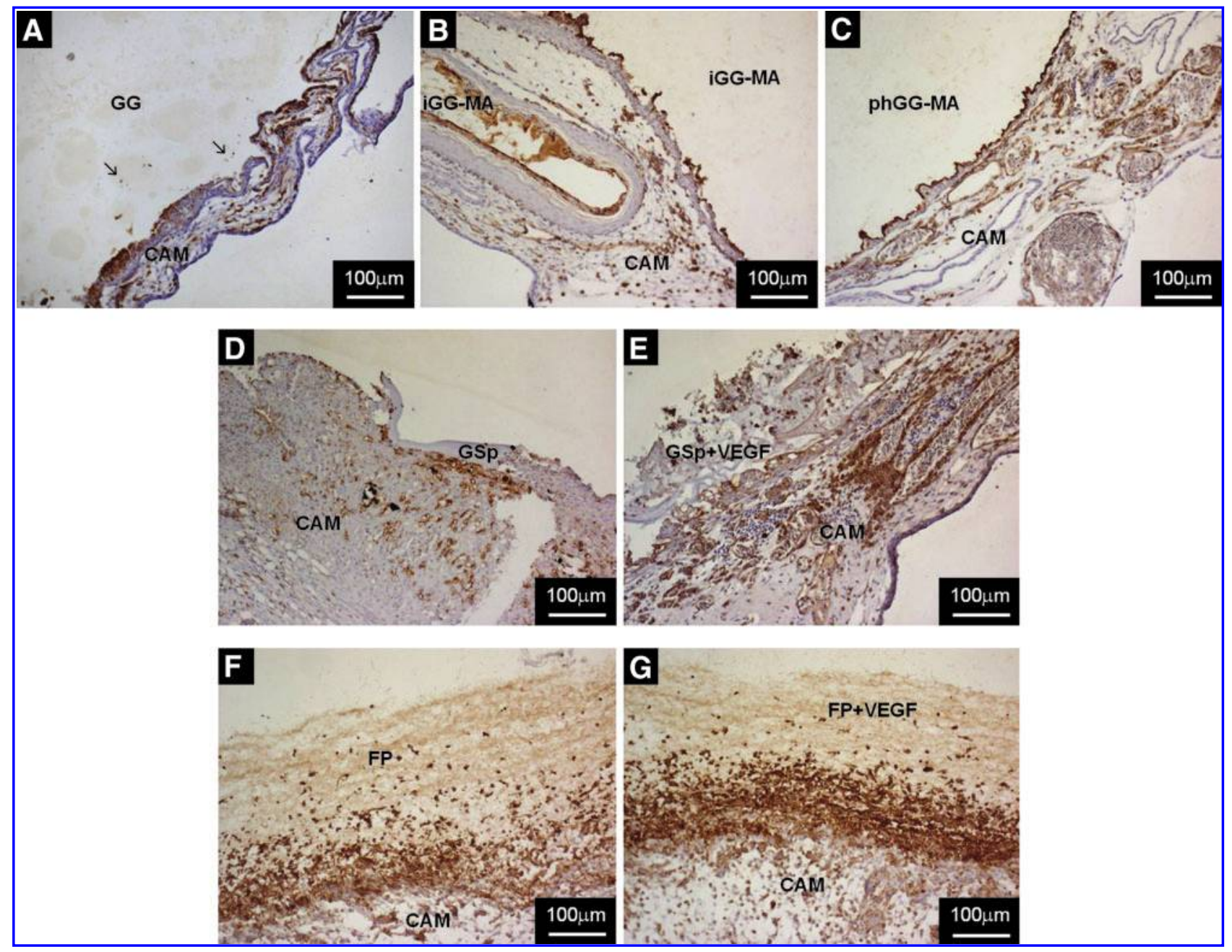

FIG. 5. Light microscopy photographs of the immunohistochemical staining of the excised CAM sagital sections corresponding to the implanted materials, after 4 days of implantation: (A) GG, (B) iGG-MA, (C) phGG-MA, (D) gelatin sponge (GSp), (E) GSp + VEGF, (F) FP, and (G) FP + VEGF. Sections were stained with SNA-lectin for endothelial cells (brown) and the images were acquired under light microscopy. The arrows indicate infiltrating cells. Color images available online at www.liebertonline.com/tea

extracellular matrix by native cells (or other implanted cells) and restoration of NP functionality. Beyond the obvious needs for obtaining NP substitutes possessing such properties, these materials should also be able to control the angiogenic process and prevent vascular invasion. Only one recent study regarding the development of a biomaterial aimed for articular cartilage and intervertebral disc regeneration demonstrated its ability to inhibit the ingrowth of blood vessels. ${ }^{17}$ As proposed by the authors, an injectable hydrogel with such capability will be extremely advantageous and promissory for regeneration of cartilaginous tissues.

For evaluating the angiogenic/antiangiogenic potential of the GG-based hydrogels, the CAM assay was initially performed after 8 days of implantation (data not shown). In this preliminary study, the GG, iGG-MA, and phGG-MA hydrogels were partially adsorbed during the assay. As a result of a prolonged period of implantation, the controls did not present a regular response and the GSp was often completely absorbed, which made the observation of the implants impossible. Moreover, some degree of variability was found between replicates and biological contamination occurred frequently, which hindered both the identification of the implantation site and the quantification of the convergent macroscopic blood vessels. To avoid such complications, we have reduced the assay duration from 8 to 4 days of implantation. This procedure reduced the possibility of biological contamination, and allowed a better identification of implants and quantification of the macroscopic blood vessels. Besides, no consensus regarding time conditions (i.e., the day of implantation or its duration) for testing biomaterials in the CAM has been reached so far. ${ }^{17,22,23}$ The materials were implanted on the CAM at day 10 of embryonic development, since between days 9 and 12 the chick membrane reaches its maximum vascularization potential. ${ }^{24}$

In the present study, it was possible to observe from the ex ovo and in ovo images that all the GG-based hydrogels are relatively stable during the period of implantation. However, the materials allowed a good integration on the CAM, with a small extent of absorption. This feature is important when considering a scaffold to be used as a temporary implant; that is, it is well integrated and stable when implanted, but is continually absorbed while being replaced by the new-formed 
tissue. The quantification of the macroscopic blood vessels converging toward the implanted materials demonstrated that the GG-based hydrogels do not differ in respect to their angiogenic capacity. The results revealed that both the methacrylate groups incorporated in the structure of GG (in the iGG-MA and phGG-MA hydrogels) and the photoinitiator used to photo-crosslink the phGG-MA hydrogel do not have a significant influence on the formation of new blood vessels. The controls of angiogenesis used were based on two different materials (i.e., GSp and FP), since no report of the most suitable control materials was found in the literature. The GSp was already used in the CAM assay as a carrier of a stimulator or inhibitor of blood vessel formation and also for the delivery of cell suspensions onto the CAM. ${ }^{18,25}$ It was shown that it is well tolerated, adheres firmly to the CAM surface, and elicits very little, if any, inflammatory reaction. A common problem associated with the use of GSp is the low stability of the material during the time of the assay, since it is rapidly absorbed. FP-based materials were already shown to stimulate angiogenesis and induce the formation of inflammatory tissue, thus serving as a positive control. ${ }^{22}$ However, in another study, this type of material served as negative control for angiogenesis. ${ }^{26}$ In a systematic study on the angiogenic and inflammatory effects of different materials (e.g., glassfiber filters, viscose and GSp, and agarose and polyacrylamide gels) implanted on the CAM, it was demonstrated that all the materials promoted an inflammatory response. ${ }^{22}$ However, they differed on the level of angiogenic induction and the quality of the cellular response. The VEGF is commonly added to the materials to induce a positive effect on angiogenesis, allowing them to function as a positive control for induction of blood vessel formation. In the present study, however, the stimulation with VEGF in GSp control did not increase the number of macroscopic blood vessels converging to the material. This fact may have resulted from the low stability of GSp, which was rapidly absorbed, and did not allow an easy identification of the material for a correct addition of VEGF after 2 days of implantation. Despite, no statistically significant differences were observed on macroscopic blood vessel formation when comparing GG-based hydrogels and GSp controls. This fact supports the idea that the tested materials do not stimulate blood vessel formation, given that the GSp is often used in the CAM assay as a carrier for testing the effect of other compounds without influencing angiogenesis. ${ }^{25}$ Moreover, the significant low number of macroscopic blood vessels observed for the GG-based materials as compared with the FP controls (positive control for angiogenesis) corroborates the hypothesis that the developed hydrogels do not have an impact on promoting angiogenesis. In brief, the results from semiquantitative analysis of macroscopic blood vessels revealed that GG-based hydrogels are nonangiogenic.

Histological analysis (H\&E staining) showed that phGGMA possesses a higher number of microscopic blood vessels as compared with iGG-MA and GG. Nevertheless, both macro- and microscopic blood vessel density was decreased as compared with GSp and FP controls. VEGF was also added to GSp and FP controls to analyze whether addition would stimulate blood vessel formation. From our results, we observe that the addition of VEGF promoted the migration of cells to the CAM and stimulated blood microvessel formation. Staining with SNA-lectin demonstrated a significant difference in blood microvessel density, and the number of recruited and infiltrated chick endothelial cells in the GGbased hydrogels was decreased as compared with GSp and FP controls (with and without VEGF stimulation). Further, the results of the histological study showed that the GGbased hydrogels do not induce the formation of inflammatory tissue, which shows that the materials are noncytotoxic.

The cellular reaction to biomaterials comprises two features: the distribution of cellular population and the type of infiltrating cells. ${ }^{22}$ In this study, we observed that iGG-MA and phGG-MA hydrogels prevented cell ingrowth and blood vessel infiltration, which shows that methacrylation of GG allowed us tuning the hydrogel permeability to cells. By its turn, the GSp and GSp + VEGF were also completely infiltrated with cells, and microscopic blood vessels in the inner part of the hydrogels were found. FP and FP+VEGF stimulated angiogenesis and induced the formation of inflammatory tissue, as observed by the presence of cells consisting mainly of macrophages, fibroblasts, and endothelial cells. As suggested in the work by Zwadlo-Klarwasser ${ }^{22}$ the angiogenic activity and inflammatory response of biomaterials is strongly dependent on the chemical composition and the physical structure of the material. In this context, angiogenesis seems to be particularly induced by rough materials, such as the FP, together with an inflammatory response (formation of granulation tissue).

In brief, the present study data revealed that the GG-based hydrogels are noncytotoxic and possess different permeability to cells. This particular feature of GG and its derivatives allowed us to envision developing NP substitutes that are nonangiogenic and have tunable cellular permeability. By means of using different formulations of GG, we can not only envision and provide enhanced control over degradation rate and mechanical properties of the hydrogels, but also control cell and blood vessel infiltration ability.

\section{Conclusions}

In this work we demonstrated that the previously developed GG-based hydrogels do not present an angiogenic effect as compared with FP controls. Results showed that GG, iGG-MA, and phGG-MA hydrogels do not differ in their ability to induce/inhibit the formation of new blood vessels. On the other hand, we have shown by histological and immunohistochemical analyses that iGG-MA and phGG-MA hydrogels are nonpermissive to the ingrowth of endothelial cells. The data presented herein also demonstrated that the materials do not elicit any acute inflammatory response. As angiogenesis is a process strongly associated with the progression of NP degeneration, the prevention of vascular invasion observed in iGG-MA and phGG-MA hydrogels could be a vital factor contributing to the regeneration of degenerated intervertebral disc tissue. Therefore, due to its advantageous and tunable physicochemical properties, and ability to control cell infiltration and blood vessel invasion, the GG-based hydrogels have a strong potential as viable NP substitutes.

\section{Acknowledgments}

The authors thank the funds provided by Portuguese Foundation for Science and Technology (FCT) through POCTI and FEDER programs (SFRH/BI/33503/2008). This work was also carried out with the support of the European 
Union funded Collaborative Project Disc Regeneration (NMP3-LA-2008-213904).

\section{Disclosure Statement}

No competing financial interests exist.

\section{References}

1. Setton, L.A., Bonassar, L., and Masuda, K. Regeneration and replacement of the intervertebral disc. In: Lanza, R., Langer, R., and Vacanti, J., eds. Principles of Tissue Engineering, 3rd Edition. Burlington: Academic Press, 2007, pp. 875-894.

2. Pereira, D.R., Silva-Correia, J., Oliveira, J.M., and Reis, R.L. Hydrogels in acellular and cellular strategies for intervertebral disc regeneration. J Tissue Eng Regen Med 2011 [Epub ahead of print]; DOI: 10.1002/term.500.

3. Chou, A.I., and Nicoll, S.B. Characterization of photocrosslinked alginate hydrogels for nucleus pulposus cell encapsulation. J Biomed Mater Res A 91A, 187, 2009.

4. Bron, J.L., Vonk, L.A., Smit, T.H., and Koenderink, G.H. Engineering alginate for intervertebral disc repair. J Mech Behav Biomed Mater 4, 1196, 2011.

5. Cloyd, J., Malhotra, N., Weng, L., Chen, W., Mauck, R., and Elliott, D. Material properties in unconfined compression of human nucleus pulposus, injectable hyaluronic acid-based hydrogels and tissue engineering scaffolds. Eur Spine J 16, 1892, 2007.

6. Reza, A.T., and Nicoll, S.B. Characterization of novel photocrosslinked carboxymethylcellulose hydrogels for encapsulation of nucleus pulposus cells. Acta Biomater 6, 179, 2010.

7. Collin, E.C., Grad, S., Zeugolis, D.I., Vinatier, C.S., Clouet, J.R., Guicheux, J.J., Weiss, P., Alini, M., and Pandit, A.S. An injectable vehicle for nucleus pulposus cell-based therapy. Biomaterials 32, 2862, 2011.

8. Roughley, P., Hoemann, C., DesRosiers, E., Mwale, F., Antoniou, J., and Alini, M. The potential of chitosan-based gels containing intervertebral disc cells for nucleus pulposus supplementation. Biomaterials 27, 388, 2006.

9. Cheng, Y.-H., Yang, S.-H., and Lin, F.-H. Thermosensitive chitosan-gelatin-glycerol phosphate hydrogel as a controlled release system of ferulic acid for nucleus pulposus regeneration. Biomaterials 32, 6953, 2011.

10. Oliveira, J.T., Martins, L., Picciochi, R., Malafaya, P.B., Sousa, R.A., Neves, N.M., Mano, J.F., and Reis, R.L. Gellan gum: a new biomaterial for cartilage tissue engineering applications. J Biomed Mater Res A 93, 852, 2010.

11. Oliveira, J.T., Santos, T.C., Martins, L., Picciochi, R., Marques, A.P., Castro, A.G., Neves, N.M., Mano, J.F., and Reis, R.L. Gellan gum injectable hydrogels for cartilage tissue engineering applications: in vitro studies and preliminary in vivo evaluation. Tissue Eng Part A 16, 343, 2010.

12. Oliveira, J.T., Gardel, L.S., Rada, T., Martins, L., Gomes, M.E., and Reis, R.L. Injectable gellan gum hydrogels with autologous cells for the treatment of rabbit articular cartilage defects. J Orthop Res 28, 1193, 2010.

13. Pereira, D.R., Silva-Correia, J., Caridade, S.G., Oliveira, J.M., Salgado, A.J., Sousa, N., Mano, J.F., and Reis, R.L. Development of gellan gum-based microparticles/hydrogel matrices for application in the intervertebral disc regeneration. Tissue Eng Part C 17, 961, 2011.

14. Silva-Correia, J., Oliveira, J.M., Caridade, S.G., Oliveira, J.T., Sousa, R.A., Mano, J.F., and Reis, R.L. Gellan gum-based hydrogels for intervertebral disc tissue-engineering applications. J Tissue Eng Regen Med 5, e97, 2011.
15. Silva-Correia, J., Oliveira, J.M., Oliveira, J.T., Sousa, R.A., and Reis, R.L. Photo-crosslinked gellan gum-based hydrogels: methods and uses thereof. WO2011/119059, Priority date: 105030 26.03.2010 PT.

16. Freemont, A.J. The cellular pathobiology of the degenerate intervertebral disc and discogenic back pain. Rheumatology $48,5,2009$.

17. Scholz, B., Kinzelmann, C., Benz, K., Mollenhauer, J., Wurst, H., and Schlosshauer, B. Suppression of adverse angiogenesis in an albumin-based hydrogel for articular cartilage and intervertebral disc regeneration. Eur Cell Mater 20, 24, 2010.

18. Ribatti, D., Nico, B., Vacca, A., and Presta, M. The gelatin sponge-chorioallantoic membrane assay. Nat Protoc 1, 85, 2006.

19. Deryugina, E., and Quigley, J. Chick embryo chorioallantoic membrane model systems to study and visualize human tumor cell metastasis. Histochem Cell Biol 130, 1119, 2008.

20. Deryugina, E.I., and Quigley, J.P. Chapter two: chick embryo chorioallantoic membrane models to quantify angiogenesis induced by inflammatory and tumor cells or purified effector molecules. Methods Enzymol 444, 21, 2008.

21. Vargas, A., Zeisser-Labouèbe, M., Lange, N., Gurny, R., and Delie, F. The chick embryo and its chorioallantoic membrane (CAM) for the in vivo evaluation of drug delivery systems. Adv Drug Deliv Rev 59, 1162, 2007.

22. Zwadlo-Klarwasser, G., Görlitz, K., Hafemann, B., Klee, D., and Klosterhalfen, B. The chorioallantoic membrane of the chick embryo as a simple model for the study of the angiogenic and inflammatory response to biomaterials. J Mater Sci Mater Med 12, 195, 2001.

23. Oates, M., Chen, R., Duncan, M., and Hunt, J.A. The angiogenic potential of three-dimensional open porous synthetic matrix materials. Biomaterials 28, 3679, 2007.

24. Borges, J., Tegtmeier, F.T., Padron, N.T., Mueller, M.C., Lang, E.M., and Stark, G.B. Chorioallantoic membrane angiogenesis model for tissue engineering: a new twist on a classic model. Tissue Eng 9, 441, 2003.

25. Ribatti, D., Vacca, A., Roncali, L., and Dammacco, F. The chick embryo chorioallantoic membrane as a model for in vivo research on anti-angiogenesis. Curr Pharm Biotechnol 1, 73, 2000.

26. Ahmadi, R., Burns, A.J., and de Bruijn, J.D. Chitosan-based hydrogels do not induce angiogenesis. I Tissue Eng Regen Med 4, 309, 2010.

Address correspondence to: Joana Silva-Correia, Ph.D. Department of Polymer Engineering 3B's Research Group-Biomaterials, Biodegradables and Biomimetics University of Minho Headquarters of the European Institute of Excellence on Tissue Engineering and Regenerative Medicine AvePark, S. Cláudio de Barco Caldas das Taipas Guimarães 4806-909 Portugal

E-mail: joana.correia@dep.uminho.pt

Received: November 8, 2011

Accepted: January 27, 2012

Online Publication Date: May 2, 2012 